\title{
Structural morphology and relaxation spectra of viscoelastic heterogeneous materials
}

\author{
Stéphan Beurthey ${ }^{\mathrm{a}}$, André Zaoui ${ }^{\mathrm{b}}$ \\ ${ }^{a}$ CTA, 16 bis av. Prieur de la Côte d'Or, F-94114 Arcueil Cedex, France \\ ${ }^{\mathrm{b}}$ Laboratoire de Mécanique des Solides, Ecole Polytechnique, CNRS, F-91128 Palaiseau Cedex, France
}

\begin{abstract}
We analytically derive the relaxation spectra of a two-phase isotropic material whose phases are isotropic Maxwell media, according to the classical and to the generalized self-consistent schemes. Whereas these spectra are continuous in both cases, they exhibit strong differences which can be associated with the different underlying morphology, either symmetrical (polycrystal-type) in the first case or asymmetrical (composite-type) in the second case. The treatment is extended to the $(N+1)$-phase model which allows us to deal with coated inclusions or with an interphase between the matrix and the inclusions: the interphase is shown to strongly modify the resultant spectrum. More general cases are then considered for different kinds of constitutive behaviour as well as for coated fibre reinforced composites. As a whole, the spectral analysis method appears to be an efficient tool for the investigation of the connection between structural morphology and the overall behaviour of viscoelastic heterogeneous materials.
\end{abstract}

spectral analysis / structural morphology / heterogeneous materials

\section{Introduction}

The influence of internal morphological characteristics on the overall behaviour of micro-inhomogeneous materials is a matter of increasing work and interest. Up to now, this topic has been mainly developed in the framework of linear elasticity (since Kröner, 1977). Basic questions such as the way to take into account the inclusion/matrix morphology of composite materials or the disordered constitution of polycrystals have been given satisfying practical answers in this case, especially through the classical self-consistent scheme (C.S.C.S., Hershey, 1954) on the one hand, for intricate morphologies where no phase plays any specific morphological role, and through the composite spheres (or cylinders) assemblage (Hashin, 1962) and the associated generalized self-consistent scheme (G.S.C.S. or three-phase model, Christensen and Lo, 1979) on the other hand, when one phase is disposed as a well-connected matrix and the other ones are dispersed in it. The latter case has been generalized in different ways, either by taking multilayered inclusions into account through so-called $(N+1)$-phase models in order to deal with coated particles or fibres (Hervé and Zaoui, 1993; Hervé and Zaoui, 1995) or by considering arbitrary composite patterns as basic units of heterogeneous materials through 'morphological pattern-based' bounding or estimating treatments (Bornert et al., 1996).

Nevertheless the generalization of such approaches to nonlinear behaviour has to overcome strong difficulties which originate in the fact that the linearized (either secant or tangent) moduli of the phases cannot be treated as uniform per phase (Zaoui, 1997). Whereas many current works are devoted to rigorous or approximate treatments of this problem, this paper proposes to explore another route within the same general field in order to enlarge the knowledge of the connection between micro-morphology and the overall behaviour of heterogeneous materials. This approach is based on the extension of known treatments for the case of linear elasticity to linear (non ageing) viscoelasticity. The difficult coupling of elasticity and viscosity, which makes 
straightforward extensions of Green's techniques inefficient due to the simultaneous occurrence of derivatives of different orders of the mechanical variables in the considered constitutive equations, can then be easily treated with the help of the Laplace transformation technique and the associated 'correspondence principle' (Mandel, 1966, Laws and McLaughlin, 1978): this principle allows one to transform an inhomogeneous viscoelastic problem into a symbolical elastic one which may have a known solution and to go back to real variables by inversion of the Laplace transformation. Such a method makes it possible to study actual viscoelastic heterogeneous materials, such as reinforced polymer matrices or polymer blends, to investigate the mechanical influence of interphases between (possibly coated) inclusions and the matrix or to serve as a basis for further extensions to nonlinear viscoelasticy or rate-dependent elastoplasticity. An additional interest can be found in the use of the spectral analysis of the overall behaviour through the derivation of the relaxation spectra of such materials which is expected to give access to significant information on the micro-morphology.

This point can be illustrated as follows: we consider a multiphase material consisting of Maxwellian constituents with local constitutive equations of the form

$$
\dot{\varepsilon}=\mathbf{a}: \sigma+\mathbf{b}: \dot{\sigma}
$$

with $\boldsymbol{\varepsilon}$ and $\boldsymbol{\sigma}$ the strain and strain tensors and $\mathbf{a}$ and $\mathbf{b}$ material constant fourth order tensors. Whereas the overall behaviour is known to be linear viscoelastic, it is no more Maxwellian due to the coupling between elasticity and viscosity and the associated 'long range memory effect'. The overall constitutive equations are expected in the form (Suquet, 1987):

$$
\dot{\mathbf{E}}=\mathbf{A}^{\mathrm{eff}}: \boldsymbol{\Sigma}+\mathbf{B}^{\mathrm{eff}}: \dot{\boldsymbol{\Sigma}}+\int_{0}^{t} \mathbf{J}(t-\tau): \dot{\boldsymbol{\Sigma}}(\tau) \mathrm{d} \tau
$$

where $\mathbf{E}$ and $\boldsymbol{\Sigma}$ are the macroscopic strain and stress tensors, $\mathbf{A}^{\text {eff }}$ and $\mathbf{B}^{\text {eff }}$ are the macroscopic (or effective) analogues of $\mathbf{a}$ and $\mathbf{b}$ and $\mathbf{J}(t-\tau)$ is an interaction kernel which reflects the complexity of the delayed mechanical interactions between the phases.

How can this kernel be represented by relaxation spectra? How does it depend on the considered morphology? What do the associated relaxation spectra look like according to this morphology? How can they be attached to a polycrystal-type or to a composite-type morphology? How does an interphase affect the shape of the relaxation spectra? What happens when coated particles or fibres are considered? These are the main questions which are addressed in the following, through the use of the C.S.C.S., of the G.S.C.S. and of the $(N+1)$-phase model for simple linear viscoelastic constituents. This is performed through the use of the Laplace(-Carson) transformation technique in order to convert the analysis of the coupling of elasticity and viscosity into the resolution of a symbolical linear elastic problem.

In the first part (Section 2), we determine the shape of the shear relaxation spectra when each phase has only one relaxation time, as a function of the morphology, by comparing the predictions of the 'classical' and the 'generalized' self-consistent models. We then consider (Section 3) the ' $(N+1)$-phase model' which is based on the configuration of a multilayered spherical inclusion surrounded by the equivalent homogeneous medium. Each phase may have several relaxation times. Thus the existence of an interphase located between the inclusions and a continuous matrix can be taken into account. The comparison of the relaxation spectra indicates an impressive 'signature' of the interface. Other applications are suggested. Finally, we report similar results for cylindrical geometry (Section 4). 


\section{Shear relaxation spectra of two-phase isotropic Maxwellian materials}

Assuming local isotropy and incompressibility for the sake of simplicity, we write the constitutive equations for each phase in the form:

$$
\dot{\mathbf{e}}=a_{i} \mathbf{s}+b_{i} \dot{\mathbf{s}}, \quad i=1,2,
$$

where $a_{i}$ and $b_{i}$ are scalar constants and $\mathbf{s}$ and $\mathbf{e}$ are the stress and strain deviators. After Laplace-Carson transformation, defined by

$$
f^{*}(p)=p \int_{0}^{\infty} f(t) \mathrm{e}^{-p t} \mathrm{~d} t
$$

we get:

$$
\mathbf{s}^{*}(p)=2 \mu_{i}^{*}(p) \mathbf{e}^{*}(p), \quad \mu_{i}^{*}(p)=\frac{p}{2 b_{i}\left(p+1 / t_{i}\right)},
$$

with $p$ the complex variable, $t_{i}=b_{i} / a_{i}$ the (single) relaxation time of phase $(i)$ with the intensity $1 / 2 b_{i}$. The two-phase material is supposed to be isotropic too. Due to incompressibility, it is completely defined by the overall shear relaxation modulus $\mu^{\mathrm{eff}}(t)$. According to the theory of spectral analysis, we are looking for this modulus in the form

$$
\mu^{\mathrm{eff}}(t)=\int_{0}^{\infty} g^{\mathrm{eff}}(\tau) \mathrm{e}^{-t / \tau} \mathrm{d} \tau
$$

where $g^{\text {eff }}(t)$ gives access to the effective shear relaxation spectrum — which reduces for each phase $(i)$ to one single line at $t=t_{i}$ with the intensity $1 / 2 b_{i}$. Two self-consistent models will be considered concurrently, the classical and the generalized ones. Owing to the correspondence principle, $\mu^{\text {eff* }}(p)$ is obtained from $\mu_{1}^{*}(p)$ and $\mu_{2}^{*}(p)$ through the same equations as those which relate $\mu^{\text {eff }}, \mu_{1}$ and $\mu_{2}$ in the elastic case. These classical quadratic equations have the form:

$$
A \cdot X^{2}+B \cdot X+C=0,
$$

where $X=\mu^{\mathrm{eff}} / \mu_{1}$. For the C.S.C.S. we have:

$$
A=1, \quad B=\frac{2-5 c}{3} \frac{\mu_{2}}{\mu_{1}}+\frac{5 c-3}{3}, \quad C=-\frac{2}{3} \frac{\mu_{2}}{\mu_{1}}
$$

with $c$ the volume fraction of phase (2). For the G.S.C.S., with phase (1) as the continuous matrix, $A, B$ and $C$ are second order polynoms in $\left(\mu_{2} / \mu_{1}\right)$ and tenth order polynoms in $c^{1 / 3}$ (see Appendix 1). For the sake of simplicity, we define $A$ such that it is non-negative for any $c$. In both cases, equation (7) has only one real positive root.

For the present viscoelastic case, we define $X^{*}(p)$ as:

$$
X^{*}(p)=\frac{\mu^{\mathrm{eff} *}(p)}{\mu_{1}^{*}(p)}
$$

Now $X^{*}(p)$ is a solution of an equation of the form (Rougier et al., 1993):

$$
A^{*} X^{* 2}+B^{*} X^{*}+C^{*}=0
$$


where $A^{*}, B^{*}$ and $C^{*}$ depend on $\mu_{2}^{*}(p) / \mu_{1}^{*}(p)$ and $c$ in the same way as $A, B$ and $C$ depend on $\mu_{2} / \mu_{1}$ and $c$. As a result, $\mu^{\text {eff* }}(p)$ can be expressed as the sum of two terms:

$$
\mu^{\mathrm{eff} *}(p)=f_{1}^{*}(p) \pm f_{2}^{*}(p), \quad f_{1}^{*}(p)=-\frac{p B^{*}}{4 b_{1}\left(p+1 / t_{1}\right) A^{*}}, \quad f_{2}^{*}(p)=\frac{p\left(B^{* 2}-4 A^{*} C^{*}\right)^{1 / 2}}{4 b_{1}\left(p+1 / t_{1}\right) A^{*}}
$$

The first term $f_{1}^{*}(p)$ is a rational fraction in $p$, so that $f_{1}(t)$ is a sum of decreasing exponential functions: two for the C.S.C.S., contributing to the spectrum by two single lines at times $t_{1}$ and $t_{2}$, but three for the G.S.C.S., contributing to the spectrum by three single lines, one at the matrix relaxation time $t_{1}$ and two other ones at times $\theta_{1}$ and $\theta_{2}$ which lie somewhere between $t_{1}$ and $t_{2}$. Note for the moment that their respective intensities may be negative according to the value of $c$.

After some reduction using (9) and (11), $f_{2}^{*}(p)$ can be written for the C.S.C.S. as:

$$
\begin{aligned}
f_{2}^{C *}(p) & =\frac{k^{C} p\left[P^{C}(p)\right]^{1 / 2}}{\left(p+1 / t_{1}\right)\left(p+1 / t_{2}\right)}, \quad P^{C}(p)=\left(p+1 / \tau_{1}\right)\left(p+1 / \tau_{2}\right), \\
k^{C} & =\frac{\sqrt{(2-5 c)^{2} b_{1}^{2}+2(6-5 c)(5 c+1) b_{1} b_{2}+(5 c-3)^{2} b_{2}^{2}}}{12 b_{1} b_{2}},
\end{aligned}
$$

where $\tau_{1}$ and $\tau_{2}$ are positive times lying between $t_{1}$ and $t_{2}$. For the G.S.C.S., we get:

$$
\begin{aligned}
f_{2}^{G *}(p) & =\frac{k^{G} p\left[P^{G}(p)\right]^{1 / 2}}{\left(p+1 / t_{1}\right)\left(p+1 / \theta_{1}\right)\left(p+1 / \theta_{2}\right)}, \\
P^{G}(p) & =\left(p+1 / \tau_{1}^{\prime}\right)\left(p+1 / \tau_{2}^{\prime}\right)\left(p+1 / \tau_{3}^{\prime}\right)\left(p+1 / \tau_{4}^{\prime}\right),
\end{aligned}
$$

where $\tau_{1}^{\prime}, \tau_{2}^{\prime}, \tau_{3}^{\prime}$ and $\tau_{4}^{\prime}$ are positive times such that $\tau_{1}^{\prime}, \tau_{2}^{\prime} \in\left[t_{1}, \theta_{1}\right]$ and $\tau_{3}^{\prime}, \tau_{4}^{\prime} \in\left[\theta_{1}, \theta_{2}\right]$ and $k^{G}$ is a constant given in Appendix 1.

We can easily derive $f_{2}(t)$ from (12) or (13) by use of the inverse Laplace-Carson transformation which is defined in the complex plane by:

$$
f_{2}(t)=\frac{1}{2 i \pi} \int_{\Delta} \frac{f_{2}^{*}(p)}{p} \mathrm{e}^{p t} \mathrm{~d} p
$$

where $\Delta$, parallel to the $y$-axis, must have all the poles and critical points of $f_{2}^{*}(p)$ on the left; it can be taken here as the $y$-axis itself since all these poles are real negatives.

The theorem of residues can be used after complementing $\Delta$ with a half-circle centered at the origin and located in the left half-plane $\operatorname{Re}(p) \leqslant 0$, with a radius $R$ growing to infinity; in addition, in order to get a uniform representation of $f_{2}^{*}(p)$, one has to define adequate cuts on the real negative axis on $\left[-1 / \tau_{1},-1 / \tau_{2}\right]$ for the C.S.C.S. and on $\left[-1 / \tau_{1}^{\prime} ;-1 / \tau_{2}^{\prime}\right]$ and $\left[-1 / \tau_{3}^{\prime} ;-1 / \tau_{4}^{\prime}\right]$, for the G.S.C.S. (figure 1 ). Suffice it now to make use of Jordan's lemma in order to get rid of the integral on the half-circle when $R \rightarrow \infty$ and we are left with $f_{2}(t)$ as a sum of some residues and integrals on the cuts: the residues are associated with decreasing exponentials, and then with single lines of the spectra, whereas the integrals on the cuts will be responsible for continuous parts of the spectra.

More precisely, we get (Rougier et al., 1993), with $t_{1}<t_{2}$ : 


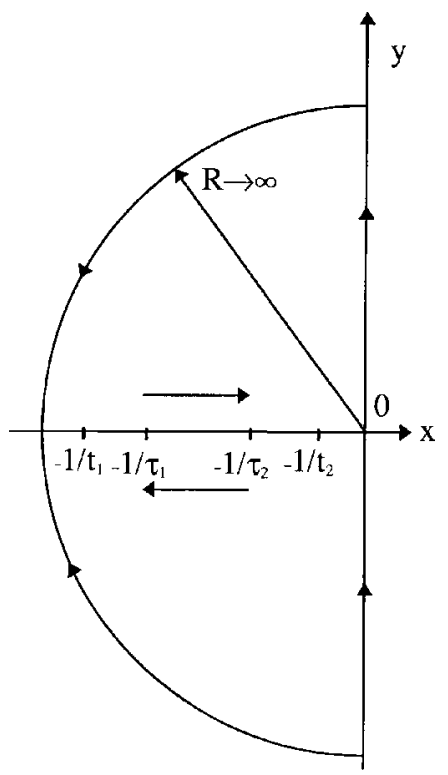

(a)

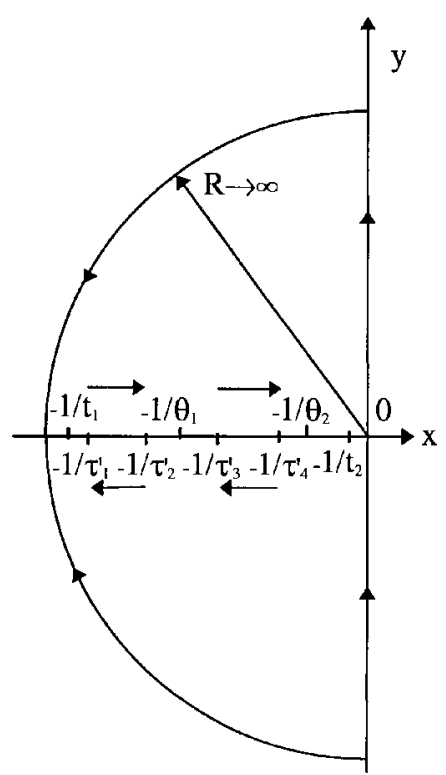

(b)

Figure 1. Integration paths used for the inversion of the Laplace transformation $\left(t_{1}<t_{2}\right)$; (a) C.S.C.S; (b) G.S.C.S.

- for the C.S.C.S.:

$f_{2}^{C}(t)= \pm \frac{k^{C}}{\pi}\left\{-\int_{-1 / \tau_{1}}^{-1 / \tau_{2}} \frac{\sqrt{-P^{C}(x)}}{\left(x+1 / t_{1}\right)\left(x+1 / t_{2}\right)} \mathrm{e}^{t x} \mathrm{~d} x-\pi \frac{\sqrt{P^{C}\left(-1 / t_{1}\right)}}{1 / t_{2}-1 / t_{1}} \mathrm{e}^{-t / t_{1}}+\pi \frac{\sqrt{P^{C}\left(-1 / t_{2}\right)}}{1 / t_{1}-1 / t_{2}} \mathrm{e}^{-t / t_{2}}\right\}$,

- for the G.S.C.S.:

$$
\begin{aligned}
f_{2}^{G}(t)= \pm \frac{k^{G}}{\pi}\{ & \int_{-1 / \tau_{1}^{\prime}}^{-1 / \tau_{2}^{\prime}} \frac{\sqrt{-P^{G}(x)}}{\left(x+1 / t_{1}\right)\left(x+1 / \theta_{1}\right)\left(x+1 / \theta_{2}\right)} \mathrm{e}^{t x} \mathrm{~d} x \\
& -\int_{-1 / \tau_{3}^{\prime}}^{-1 / \tau_{4}^{\prime}} \frac{\sqrt{-P^{G}(x)}}{\left(x+1 / t_{1}\right)\left(x+1 / \theta_{1}\right)\left(x+1 / \theta_{2}\right)} \mathrm{e}^{t x} \mathrm{~d} x \\
& +\pi \frac{\sqrt{P^{G}\left(-1 / t_{1}\right)}}{\left(1 / \theta_{1}-1 / t_{1}\right)\left(1 / \theta_{2}-1 / t_{1}\right)} \mathrm{e}^{-t / t_{1}}-\pi \frac{\sqrt{P^{G}\left(-1 / \theta_{1}\right)}}{\left(1 / t_{1}-1 / \theta_{1}\right)\left(1 / \theta_{2}-1 / \theta_{1}\right)} \mathrm{e}^{-t / \theta_{1}} \\
& \left.+\pi \frac{\sqrt{P^{G}\left(-1 / \theta_{2}\right)}}{\left(1 / t_{1}-1 / \theta_{2}\right)\left(1 / \theta_{2}-1 / \theta_{1}\right)} \mathrm{e}^{-t / \theta_{2}}\right\} .
\end{aligned}
$$

With help of the change of variable $\tau=-1 / x$, (15) and (16) can be put in the standard form (6) and yield the expected spectra after addition to $f_{1}(t)$ and determination of the front sign in (15) and (16) according to the condition $\mu^{\text {eff }}(0)=\lim _{|p| \rightarrow \infty}\left(\mu^{\text {eff }}(p)\right)>0$. The resulting spectra consist of a continuous part and discrete lines whose intensity can be checked to be always positive or null.

For the C.S.C.S. we get one continuous spectrum defined by the function

$$
g^{C}(\tau)=\frac{k^{C} t_{1} t_{2}}{\pi \sqrt{\tau_{1} \tau_{2}}} \frac{\sqrt{\left(\tau-\tau_{1}\right)\left(\tau_{2}-\tau\right)}}{\tau\left(\tau-t_{1}\right)\left(t_{2}-\tau\right)}, \quad \tau \in\left[\tau_{1}, \tau_{2}\right],
$$


and one or two discrete lines located at $\tau=t_{1}$ with the intensity $(3-5 c) / 6 b_{1}$ for $c \leqslant 3 / 5$ and at $\tau=t_{2}$ with the intensity $(5 c-2) / 6 b_{2}$ for $c \geqslant 2 / 5$.

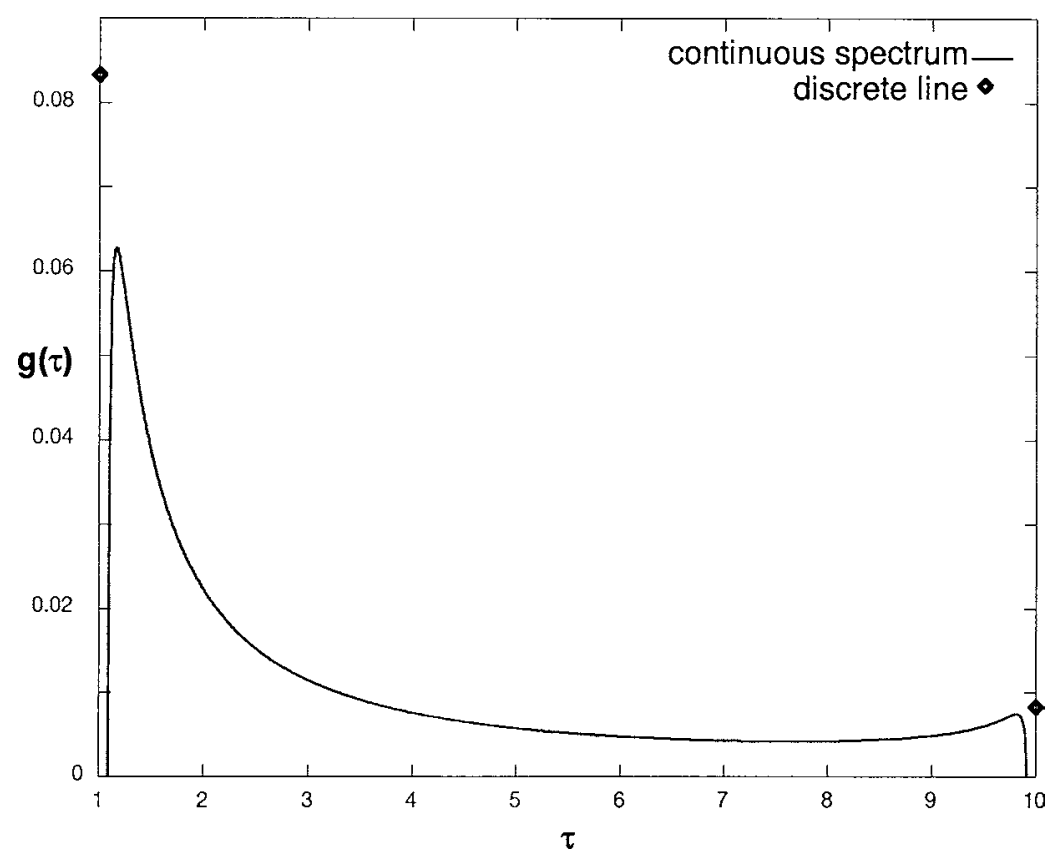

(a)

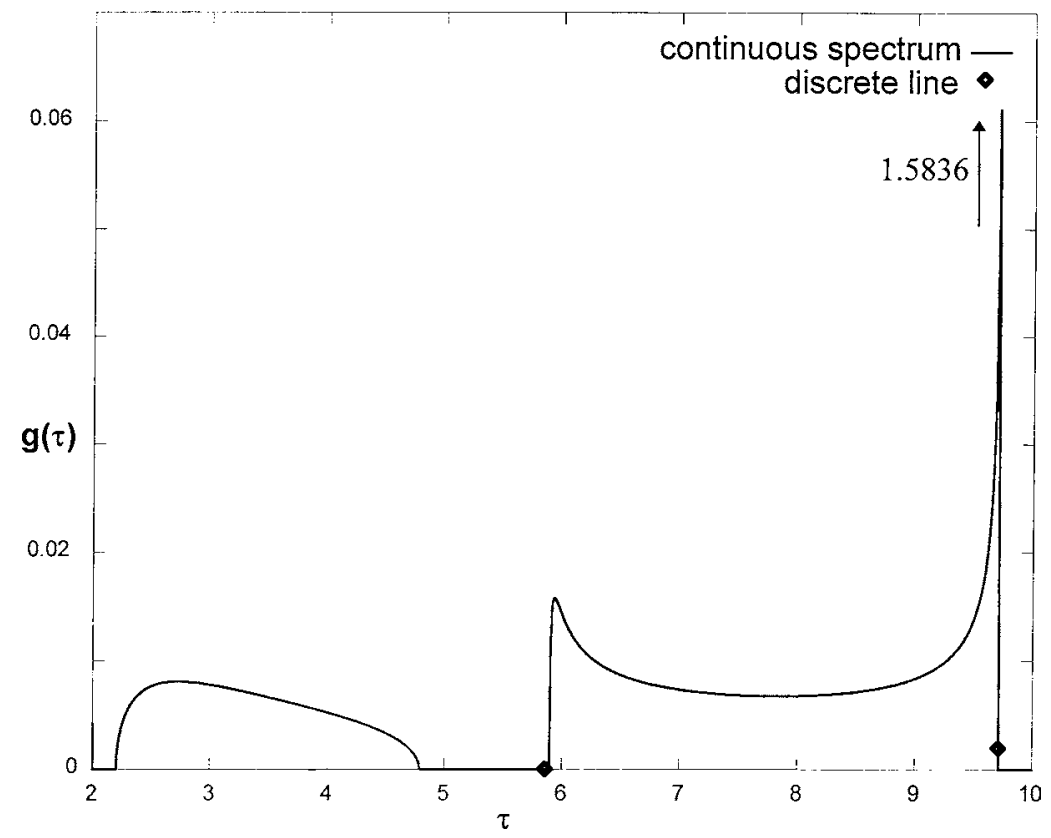

(b)

Figure 2. Comparison of the relaxation spectra as derived from the two considered models, in the case $c=0.5 ; b_{1}=1 ; b_{2}=10 ; t_{1}=1 ; t_{2}=10$; (a) classical self-consistent model; (b) generalized self-consistent model. 
For the G.S.C.S., the continuous spectrum is divided into two parts defined by

$$
\begin{gathered}
g^{G}(\tau)=\frac{\epsilon(\tau) k^{G} t_{1} \theta_{1} \theta_{2}}{\pi \sqrt{\tau_{1}^{\prime} \tau_{2}^{\prime} \tau_{3}^{\prime} \tau_{4}^{\prime}} \frac{\sqrt{\left(\tau-\tau_{1}^{\prime}\right)\left(\tau-\tau_{2}^{\prime}\right)\left(\tau-\tau_{3}^{\prime}\right)\left(\tau_{4}^{\prime}-\tau\right)}}{\tau\left(\tau-t_{1}\right)\left(\theta_{1}-\tau\right)\left(\theta_{2}-\tau\right)},} \\
\tau \in\left[\tau_{1}^{\prime}, \tau_{2}^{\prime}\right] \cup\left[\tau_{3}^{\prime}, \tau_{4}^{\prime}\right], \epsilon(\tau)=1 \text { if } \tau \in\left[\tau_{1}^{\prime}, \tau_{2}^{\prime}\right], \epsilon(\tau)=-1 \text { if } \tau \in\left[\tau_{3}^{\prime}, \tau_{4}^{\prime}\right] .
\end{gathered}
$$

Additional single lines may exist or not at $t_{1}, \theta_{1}$ and $\theta_{2}$ depending on the volume fraction $c$ and on the parameters $t_{1}, t_{2}, b_{1}$ and $b_{2}$.

Illustrative examples are reported in figure $2 a$ and $2 b$ for the C.S.C.S. and the G.S.C.S. respectively, for identical values of the material constants $c, t_{1}, t_{2}, b_{1}$ and $b_{2}$ so as to stress the influence of the sole morphology.

Other examples would lead to similar conclusions, namely:

- for both models, the resulting spectra are definitely different from Maxwell-type spectra, which would reduce to discrete lines: the continuous part reflects mainly the 'long-range memory effect' and the complicated underlying viscoelastic interactions between the phase domains as expressed, according to the self-consistent scheme, by the (simple or composite) inclusion/matrix interactions;

- in addition, the material structural morphology is shown to have a prominent influence on the shape of the continuous spectrum: whereas the symmetric morphology associated with the C.S.C.S. leads to an unbroken continuous spectrum, the composite-type morphology which is accounted for by the G.S.C.S. makes this continuous spectrum split into two separate parts and enhances the mechanical role of the matrix phase. So, these qualitative differences of the spectrum shapes, which are much more visible than the differences of the relaxation functions or of the Cole-Cole diagrams (Rougier, 1994), can be suggested to be used as 'morphological discriminators' when analysing experimental data.

\section{Generalized Maxwellian bodies: $(N+1)$-phase self-consistent modelling}

The foregoing analysis may be extended in two (possibly combined) directions: first, more general linear constitutive equations may be considered so as to deal with generalized Maxwellian constituents which are still isotropic but can be compressible and exhibit a finite number of relaxation times; second, the $(N+1)$-phase self-consistent model may be used by replacing the two-phase composite sphere of the G.S.C.S. by a composite sphere with an arbitrary number of concentric shells with different moduli. Such a model has already been defined in the case of linear elasticity (Hervé and Zaoui, 1993) so that its extension to linear viscoelasticity is straightforward: it is proposed here in the context of spectral analysis in order to emphasize, as it has been done above, the influence of the material structural morphology on the overall response.

The corresponding morphology is related to the dispersion of multiply coated inclusions in a continuous matrix, including the important case of interphases located between the matrix and the inclusions, since the difficult study of the characteristics and role of such interphases is a matter of intensive research in the field of polymer science. In addition, the $(N+1)$-phase self-consistent scheme can be taken as a simplified model when studying nonlinear composites by approximating the non uniformity of the phase secant or tangent moduli by a discretized radial variation (Bornert et al., 1994; Beurthey, 1997).

The following treatment is restricted to linear viscoelasticity: it is concerned with the spectral analysis of the $(N+1)$-phase self-consistent model consisting of an $N$-layered spherical inclusion embedded in an infinite matrix made of the searched homogenized material. Each phase has an isotropic (generalized)-Maxwellian behaviour with several relaxation times. We are looking for the overall relaxation spectra, both for the shear and the bulk moduli, with special attention paid to the case of a two-phase material with a third phase (the 
'interphase') located between the inclusions and the matrix. The mathematical treatment is a straightforward extension of the preceding one, with some additional help of numerical computations (Beurthey, 1997).

\subsection{Shear relaxation spectra}

For the sake of simplicity, we restrict ourselves to phases $(i)$ with a constant Poisson ratio $v_{i}(i=1, N)$. Their Laplace-Carson-transformed shear modulus $\mu_{i}^{*}(p)$ reads:

$$
\mu_{i}^{*}(p)=\frac{p}{2 b_{i}^{1}\left(p+1 / t_{i}^{1}\right)}+\cdots+\frac{p}{2 b_{i}^{n_{i}}\left(p+1 / t_{i}^{n_{i}}\right)}=\frac{p N_{i}^{*}(p)}{\left(p+1 / t_{i}^{1}\right) \cdots\left(p+1 / t_{i}^{n_{i}}\right)},
$$

where $N_{i}^{*}(p)$ is a polynom of degree $\left(n_{i}-1\right)$ in $p$. Through the use of the correspondence principle, the overall shear modulus $\mu^{\operatorname{eff}(N) *}(p)$ is calculated from the known quadratic equation of the elastic problem (Hervé and Zaoui, 1993) after replacing real moduli by transformed complex ones (see Appendix 2):

$$
A_{N}^{*}(p) X^{* 2}+B_{N}^{*}(p) X^{*}+C_{N}^{*}(p)=0, \quad X^{*}=\mu^{* \operatorname{eff}(N)}(p) / \mu_{N}^{*}(p),
$$

where $A_{N}^{*}(p), B_{N}^{*}(p)$ and $C_{N}^{*}(p)$ depend on $\mu_{i}^{*}(p), v_{i}$ and on the external radii $R_{i}$ of the shells $(i)$ located between $R_{i-1}$ and $R_{i}$ (figure $A 1$ ). Note that some relaxation times may be common to two adjacent shells $(i)$ and $(i+1)$ : let $j_{i, i+1}$ be the number of such values. The polynoms $A_{N}^{*}(p), B_{N}^{*}(p)$ and $C_{N}^{*}(p)$ are then of degree $d_{N}$ in $p$, with $d_{N}$ given by:

$$
d_{N}=2 \sum_{i=1}^{N-1}\left(n_{i}+n_{i+1}-j_{i, i+1}-1\right) .
$$

Except for these differences, the treatment is quite similar to the preceding one. Instead of (11), we have to set:

$$
\begin{gathered}
\mu^{\mathrm{eff}(N) *}(p)=f_{1}^{(N) *}(p) \pm f_{2}^{(N) *}(p), \quad f_{1}^{(N) *}(p)=-\frac{B_{N}^{*}(p) \mu_{N}^{*}(p)}{2 A_{N}^{*}(p)} \\
f_{2}^{(N) *}(p)=\frac{\left[B_{N}^{*}(p)^{2}-4 A_{N}^{*}(p) C_{N}^{*}(p)\right]^{1 / 2} \mu_{N}^{*}(p)}{2 A_{N}^{*}(p)}
\end{gathered}
$$

In all our numerical calculations, the polynom $A_{N}^{*}(p)$ happened to have $d_{N}$ real negative roots $\left(-1 / \theta_{k}\right)$ with $\theta_{k} \in\left[t^{\mathrm{min}}, t^{\mathrm{max}}\right]$, where $t^{\mathrm{min}}$ and $t^{\mathrm{max}}$ are the smallest and the largest relaxation times of the constituent phases, so that the rational fraction $f_{1}^{(N) *}(p)$ can be decomposed into $\left(d_{N}+n_{N}\right)$ simple elements: thus the original $f_{1}^{(N)}(t)$ is a sum of $\left(d_{N}+n_{N}\right)$ decreasing exponential functions with either positive or negative amplitudes, depending on the material parameters and phase volume fractions. As for $f_{2}^{(N) *}(p)$, we also found numerically that the polynom $\left[B_{N}^{*}(p)^{2}-4 A_{N}^{*}(p) C_{N}^{*}(p)\right]$ has $2 d_{N}$ real negative roots $\left(-1 / \tau_{k}^{\prime}\right)$ with $\tau_{k}^{\prime} \in\left[t^{\min }, t^{\max }\right]$ and no pole inserted between two consecutive roots $\left(\tau_{2 j-1}^{\prime}, \tau_{2 j}^{\prime}\right)$, so that we can write:

$$
\begin{gathered}
f_{2}^{(N) *}(p)=\mu_{N}^{*}(p) \frac{K^{(N)} \sqrt{P^{(N) *}(p)}}{\left(p+1 / \theta_{1}\right) \cdots\left(p+1 / \theta_{d_{N}}\right)}, \\
K^{(N)}=\frac{\sqrt{\left(B_{N}^{*}(0)^{2}-4 A_{N}^{*}(0) C_{N}^{*}(0)\right) \tau_{1}^{\prime} \cdots \tau_{2 d_{N}}^{\prime}}}{2 A_{N}^{*}(0) \theta_{1} \cdots \theta_{d_{N}}}, \\
P^{(N) *}(p)=\left(p+1 / \tau_{1}^{\prime}\right) \cdots\left(p+1 / \tau_{2 d_{N}}^{\prime}\right) .
\end{gathered}
$$




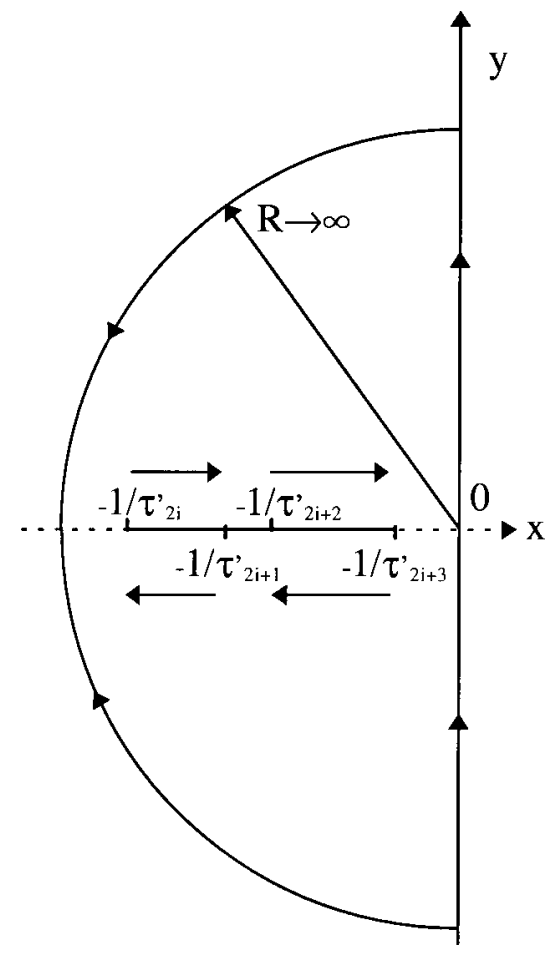

(a)

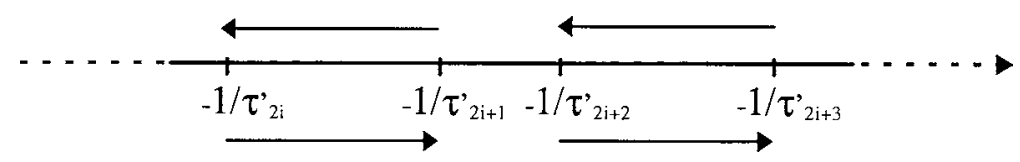

(b)

Figure 3. (a) Integration path used for the Laplace-Carson inversion; (b) roots location for $\left(1 / t_{i}<1 / t_{i+1}\right)$.

Here again, we have to define $d_{N}$ cuts on the real negative axis (figure 3 ) in view of a uniform representation of the square root in (23).

The same mathematical treatment as above leads to the following expression of $f_{2}^{(N)}(t)$ :

$$
\begin{aligned}
f_{2}^{(N)}(t)= \pm \frac{K^{(N)}}{\pi}\{ & \left.\sum_{j=1}^{d_{N}}(-1)^{j+1} \int_{-1 / \tau_{2 j-1}^{\prime}}^{-1 / \tau_{2 j}^{\prime}} \frac{\sqrt{-P^{(N)}(x)} \mu_{N}^{*}(x)}{\left(x+1 / \theta_{1}\right) \cdots\left(x+1 / \theta_{d_{N}}\right.}\right) \mathrm{e}^{t x} \mathrm{~d} x \\
& +\sum_{i=1}^{N} \frac{\pi \sqrt{-P^{(N)}\left(-1 / t_{i}^{n_{N}}\right)} \mathrm{e}^{-t / t_{i}^{n_{N}}}}{2 b_{i}^{n_{N}}\left(1 / \theta_{1}-1 / t_{i}^{n_{N}}\right) \cdots\left(1 / \theta_{d_{N}}-1 / t_{i}^{n_{N}}\right)} \\
& \left.+\sum_{j=1}^{d_{N}} \frac{(-1)^{j} \pi \sqrt{-P^{(N)}\left(-1 / \tau_{j}^{\prime}\right)} \mu_{N}^{*}\left(\tau_{j}^{\prime}\right) \mathrm{e}^{-t / \tau_{j}^{\prime}}}{\left(1 / \tau_{1}^{\prime}-1 / \tau_{j}^{\prime}\right) \cdots\left(1 / \tau_{j-1}^{\prime}-1 / \tau_{j}^{\prime}\right)\left(1 / \tau_{j+1}^{\prime}-1 / \tau_{j}^{\prime}\right) \cdots\left(1 / \tau_{d_{N}}^{\prime}-1 / \tau_{j}^{\prime}\right)}\right\}
\end{aligned}
$$

with the sign \pm chosen according to the condition $\mu^{\text {eff }}(0)=\lim _{|p| \rightarrow \infty}\left(\mu^{\text {eff }}(p)\right)>0$. After adding $f_{1}^{(N)}(t)$ and $f_{2}^{(N)}(t)$ and operating in (24) the change of variable $\tau=-1 / x, \mu^{\operatorname{eff}(N)}(t)$ can be put in the standard form (6). We get $\left(d_{N}+n_{N}\right)$ discrete lines with a positive or null amplitude at times $\theta_{j}\left(j \in\left[1, d_{N}\right]\right)$ and $t_{i}^{n_{N}}\left(i \in\left[1, n_{N}\right]\right)$, the latter ones corresponding to the matrix phase $(N)$, and a continuous spectrum split into $d_{N}$ separate parts 
on the intervals $\left[\tau_{2 j-1}^{\prime}, \tau_{2 j}^{\prime}\right]\left(j \in\left[1, d_{N}\right]\right)$ with the amplitude:

$$
\begin{aligned}
& g^{(N)}(\tau)=\left|\frac{K^{(N)} \theta_{1} \cdots \theta_{d_{N}}}{\pi \sqrt{\tau_{1}^{\prime} \cdots \tau_{2 d}^{\prime}}} \frac{\sqrt{-\left(\tau-\tau_{1}^{\prime}\right) \cdots\left(\tau-\tau_{2 d_{N}}^{\prime}\right)}}{\tau\left(\tau-\theta_{1}\right) \cdots\left(\tau-\theta_{d_{N}}\right)} \sum_{k=1}^{n_{N}} \frac{t_{N}^{k}}{2 b_{N}^{k}\left(\tau-t_{N}^{k}\right)}\right|, \\
& \tau \in\left[\tau_{1}^{\prime}, \tau_{2}^{\prime}\right] \cup \cdots \cup\left[\tau_{2 j-1}^{\prime}, \tau_{2 j}^{\prime}\right] \cup \cdots \cup\left[\tau_{2 d_{N}-1}^{\prime}, \tau_{2 d_{N}}^{\prime}\right] .
\end{aligned}
$$

Note that in (25) too the matrix phase $(N)$ plays a prominent role with respect to the other ones through the term $\left(\sum_{k=1}^{n_{N}} \frac{t_{N}^{k}}{2 b_{N}^{k}\left(\tau-t_{N}^{k}\right)}\right)$.

Now again, it is obvious that the overall behaviour is no more a (generalized) Maxwellian one since the long range memory effect leads to continuous relaxation spectra. When one relaxation time is added to some phase, the number of separate parts of the continuous spectrum may be modified through the modification of $d_{N}$ and the shape of the whole spectrum may be completely changed. When one Maxwellian layer is added, this gives rise to two additional parts of the continuous spectrum and to two new lines. Among many possible applications, we have chosen two simple illustrative examples:

- on figure 4, we use the three-phase self-consistent scheme $(N=2)$ for the case already treated on figure $2 b$ but we give an additional relaxation time to each phase. We find six (instead of two) separate parts for the continuous spectrum and six (instead of three) discrete lines with a non zero amplitude; it turns out that, if such a spectrum was got from experiment, it could be used to derive the total number of relaxation times only if the number of phases and their inclusion/matrix-type morphology is known.

- on figure 5, we still start from the same example treated on figure $2 b$ but we transform a small part of the matrix phase into a different Maxwellian material inserted between the matrix and the inclusion as

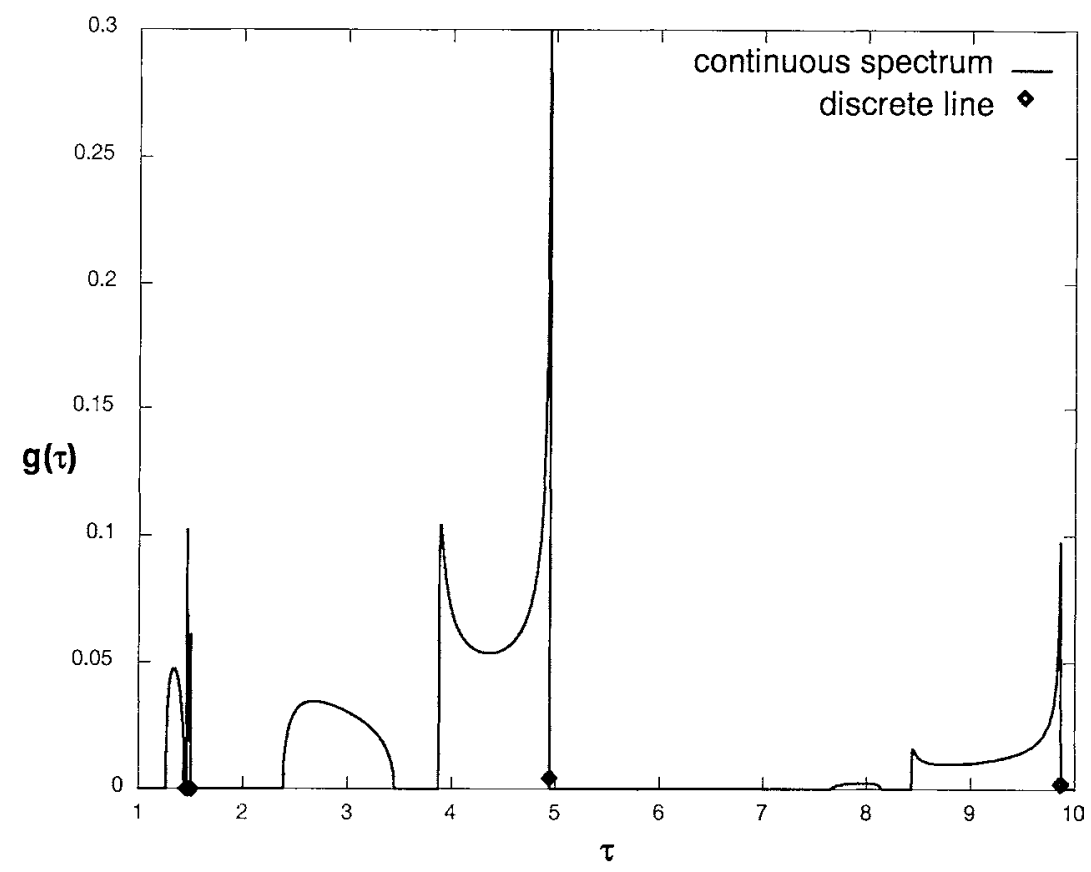

Figure 4. Shear relaxation spectrum derived from the 3-phase model for phases with two relaxation times: $v_{1}=v_{2}=0.5 ; c=0.5 ; b_{1}^{1}=10 ; b_{1}^{2}=5$; $b_{2}^{1}=1 ; b_{2}^{2}=2 ; t_{1}^{1}=10 ; t_{1}^{2}=5 ; t_{2}^{1}=1 ; t_{2}^{2}=2$. 


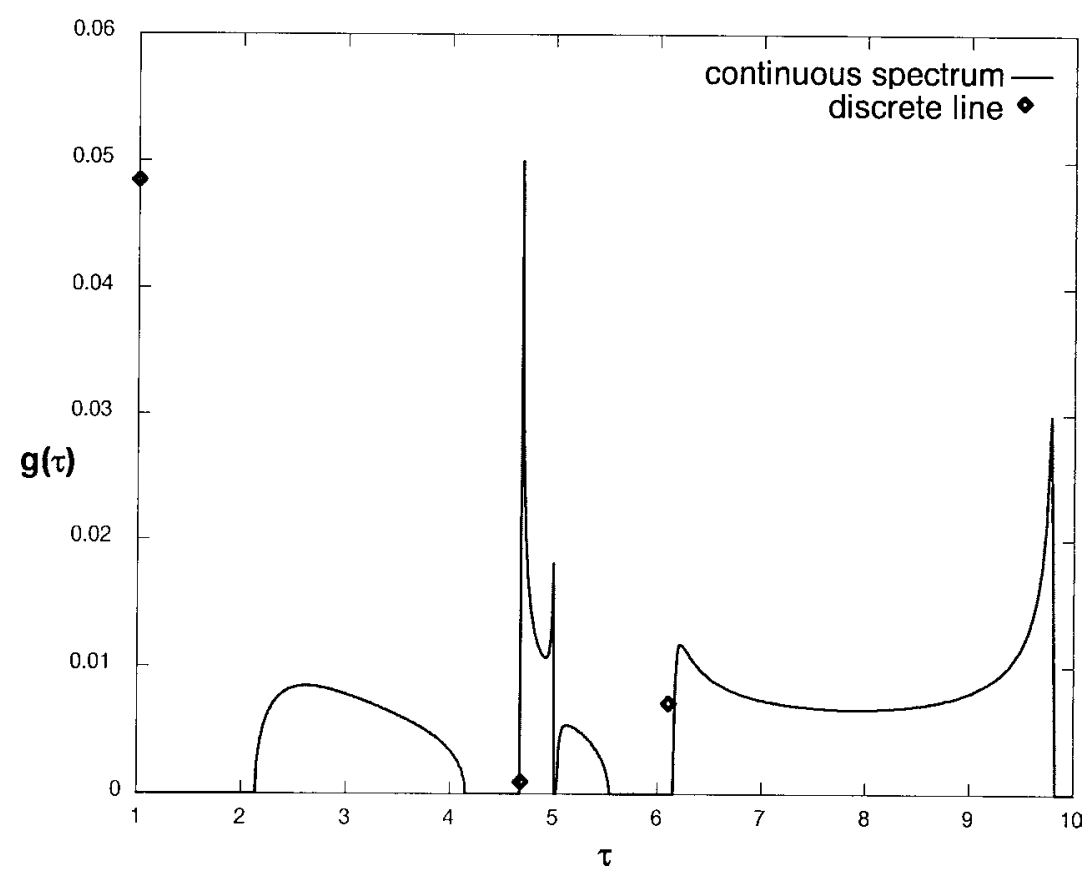

Figure 5. Shear relaxation spectrum derived from the 4-phase self consistent model, in the case of an interphase: $v_{i}=0.5 ; c_{1}=0.5 ; c_{2}=0.015$; $c_{3}=0.485 ; b_{1}=t_{1}=10 ; b_{2}=t_{2}=5 ; b_{3}=t_{3}=1$.

a thin interphase layer, with the volume fraction $c_{2}=1.5 \%$ and material constants intermediate between those of the two main phases. According to our model (with $N=3$ ), the relaxation spectrum is modified drastically, exhibiting a well-identified signature of the interphase, whereas at the same time the overall resulting creep or relaxation functions would be almost unchanged.

Here again, we can suggest from these illustrations that the spectral analysis of available experimental data on viscoelastic materials could provide useful information on the constitutive micromorphology of these materials when it is derived in connection with micromechanical models such as the one proposed hereabove.

\subsection{Bulk relaxation spectra}

The bulk relaxation spectra may be derived much more easily since, in the elastic case (Hervé and Zaoui, 1993), the overall bulk modulus $k^{\operatorname{eff}(N)}$ is given in closed form instead of as the root of a quadratic equation. From that, it can be inferred that the resulting spectra in the viscoelastic case reduce to discrete lines when the constituent phases are (generalized)-Maxwellian bodies. When Poisson's ratios are constant in time, we start from the relation:

$$
k_{i}^{*}(p)=\frac{2\left(1+v_{i}\right)}{3\left(1-2 v_{i}\right)} \mu_{i}^{*}(p), \quad i \in[1, N] .
$$

Through the use of the elastic solution, of the correspondence principle and of the expression (19) for the shear moduli, we easily find (see Appendix 2) the Laplace-Carson-transformed effective bulk modulus $k^{\text {eff }(N) *}(p)$ in the form of the rational fraction

$$
k^{\mathrm{eff}(N) *}(p)=\frac{S^{(N) *}(p)}{G^{(N) *}(p)} \mu_{N}^{*}(p),
$$




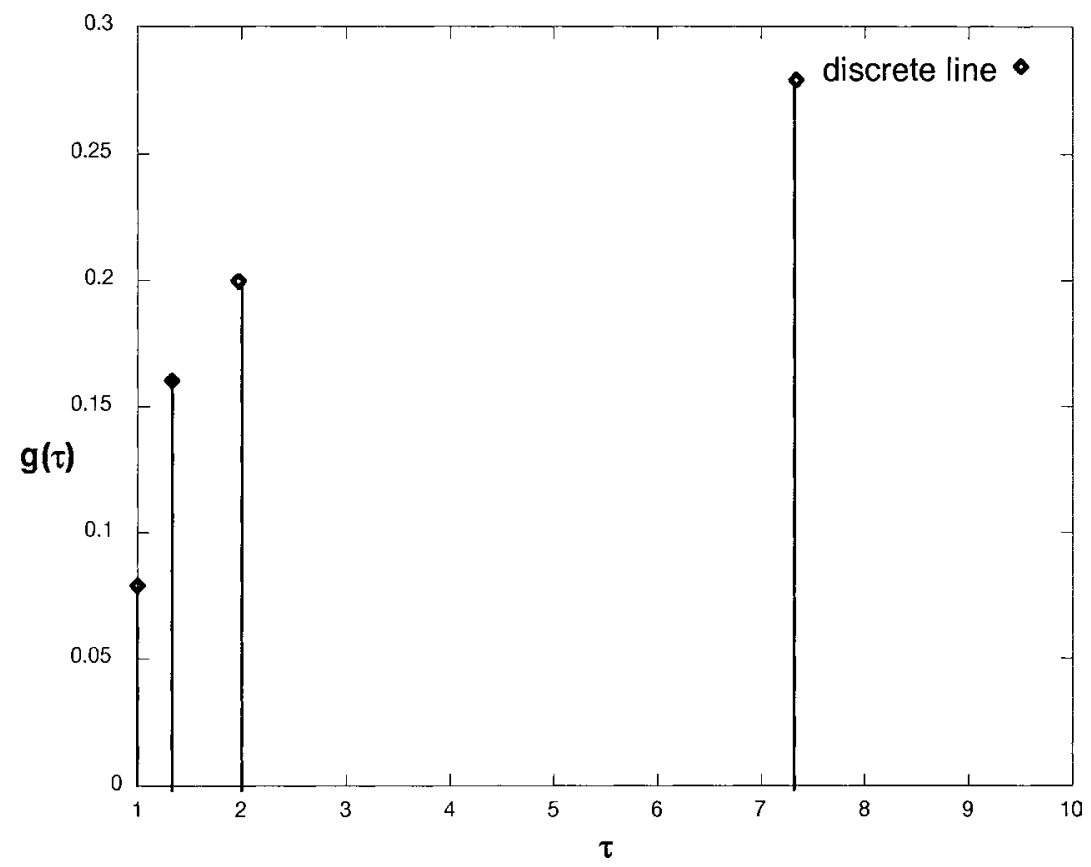

Figure 6. Bulk relaxation spectrum of bulk modulus from the 5-phase self-consistent model $\left(c_{1}=0.5 ; c_{2}=0.165 ; c_{3}=0.2 ; c_{4}=0.135 ; t_{1}=b_{1}=10\right.$; $\left.t_{2}=2 ; t_{3}=1.5 ; t_{4}=b_{4}=1 ; b_{2}=b_{3}=1\right)$.

where the polynoms $S^{(N) *}(p)$ and $G^{(N) *}(p)$ have the degree $d_{N} / 2$ in $p$. Numerical computations show that $G^{(N) *}(p)$ has $d_{N} / 2$ negative real roots $\left(-1 / \xi_{\ell}\right), \ell \in\left[1, d_{N} / 2\right]$, with $\xi_{\ell} \in\left[t^{\min }, t^{\max }\right]$. Consequently we have:

$$
k^{\operatorname{eff}(N)}(t)=\sum_{k=1}^{n_{N}} F_{k} \mathrm{e}^{-t / t_{N}^{k}}+\sum_{\ell=1}^{d_{N} / 2} E_{\ell} \mathrm{e}^{-t / \xi_{\ell}}
$$

with $F_{k}, E_{\ell}>0$. Thus the bulk relaxation spectrum reduces to discrete lines, as illustrated in figure 6 for a 5-phase model with four Maxwellian phases. Note that, for the bulk modulus only, the overall behaviour is a generalized Maxwellian one, but with relaxation times which, except for the matrix phase, do not coincide with those of the constituents.

\section{Transverse shear relaxation spectra according to the ' $(N+1)$-phase cylindrical self-consistent model'}

A straightforward extension of the foregoing analysis may be performed similarly to what has been done already in elasticity (Hervé and Zaoui, 1995) by dealing with circular cylindrical instead of spherical geometries through the ' $(N+1)$-phase cylindrical self-consistent scheme' so as to consider multiply coated fibre- (instead of particle-) reinforced composites with phases obeying a transversely isotropic (generalized) Maxwellian behaviour (which of course includes the case of linear elasticity for elastic fibres). Since the method is unchanged, we only give in Appendix 3 the new expression of the polynoms $A_{N}^{*}(p), B_{N}^{*}(p)$ and $C_{N}^{*}(p)$ for the cylindrical geometry when the fibres lie along $x_{1}$ from which, through Equations (20)-(25), one can easily derive the shear relaxation spectra for the transverse shear modulus $\mu_{23}^{\operatorname{eff}(N)}(t)$ when the Poisson ratios $v_{i}=C_{23}^{i}(t) /\left[C_{23}^{i}(t)+C_{22}^{i}(t)\right]$ actually do not depend on time $t$ (here $\mathbf{C}^{i}(t)$ is the fourth order relaxation tensor 
of phase $(i)$ ). The mathematical structure of these equations indicates that split continuous spectra will be obtained for the transverse shear modulus, whereas discrete lines only are to be expected for the four other moduli, as already known for the case $N=2$ (Bourgeois et al., 1996).

\section{Conclusion}

The foregoing analysis gives an additional unambiguous illustration of the importance of the 'long range memory effect' in viscoelastic heterogeneous materials, resulting in the fact that the overall behaviour is generally much more complex than the one of the constituents; moreover it leads to suggest to experimentalists in the field of polymer blends and reinforced polymers to make efforts in order to extract the relaxation spectra from their experimental data so as to get useful informations on the micromorphology of their materials (including the question of the presence of an interphase). Since a direct investigation of such spectra presently seems to be out of reach, this could be done by numerical deconvolution treatments applied to experimental creep/relaxation or complex moduli data.

In addition, the hereabove defined linear viscoelastic $(N+1)$-phase model can be used for a simplified treatment of the nonlinear behaviour of polymer blends (Beurthey, 1997) or composites, in a way similar to what has already been done in the field of elastoplasticity (Bornert et al., 1994; Zaoui, 1997).

\section{Appendix 1}

- The incompressible G.S.C.S.

Coefficients $A, B$ abd $C$ in (7) for the elastic incompressible G.S.C.S. (Hervé and Zaoui, 1990) read:

$$
\begin{aligned}
A= & 4\left[3(\beta-1) c-\eta_{3}\right]\left(\eta_{1} c^{7 / 3}-2 \eta_{2}\right)-126 \eta_{2}(\beta-1) c\left(1-c^{2 / 3}\right)^{2}, \\
B= & 6 \eta_{1}(\beta-1) c^{10 / 3}+8 \eta_{1} \eta_{3} c^{7 / 3}-64.5 \eta_{2}(\beta-1) c+1.5 \eta_{2} \eta_{3}+252 \eta_{2}(\beta-1) c\left(1-c^{2 / 3}\right)^{2}, \\
C= & -\left[4.5(\beta-1) c+\eta_{3}\right]\left(\eta_{1} c^{7 / 3}+9.5 \eta_{2}\right)-126 \eta_{2}(\beta-1) c\left(1-c^{2 / 3}\right)^{2} \\
& \text { with } \eta_{1}=9.5(\beta-1) ; \eta_{2}=9.5 \beta+8 ; \eta_{3}=3 \beta+4.5 ; \beta=\mu_{2} / \mu_{1} .
\end{aligned}
$$

- Shear relaxation spectrum (G.S.C.S.)

Coefficient $k^{G}$ in (13) reads:

$$
\begin{aligned}
& k^{G}=\frac{15 \sqrt{\left(u-\alpha w+\alpha^{2} y\right)^{2}-4704 c^{10 / 3}(\alpha-1)^{2}(16 \alpha+19)^{2}}}{16 b_{1}^{2}\left\{6[2(c-1)-\alpha(2 c+3)]\left[19\left(c^{7 / 3}-1\right)-\alpha\left(19 c^{7 / 3}+16\right)\right]-63(19+16 \alpha)(1-\alpha) c\left(1-c^{2 / 3}\right)^{2}\right\}} \\
& \quad \text { with } u=38\left[8 c^{10 / 3}-7-(1+14 \sqrt{6}) c\right] ; w=608 c^{10 / 3}+623-6(1+14 \sqrt{6}) c, \\
& \quad y=16\left[19 c^{10 / 3}-21+2(1+14 \sqrt{6}) c\right] ; \alpha=b_{2} / b_{1} .
\end{aligned}
$$

\section{Appendix 2}

\section{- Shear relaxation spectra}

In (19), we suppose the $j$ first relaxation times of phases $(i)$ and $(i+1)$ to be equal $\left(j=j_{i, i+1}\right)$. Coefficients $A_{N}^{*}(p), B_{N}^{*}(p)$ and $C_{N}^{*}(p)$ in (20) are then given by: 


$$
\begin{aligned}
A_{N}^{*}(p)= & 4 R_{N}^{10}\left(1-2 v_{N}\right)\left(7-10 v_{N}\right) Z_{12}^{*}+20 R_{N}^{7}\left(7-12 v_{N}-8 v_{N}^{2}\right) Z_{42}^{*}+12 R_{N}^{5}\left(1-2 v_{N}\right)\left(Z_{14}^{*}-7 Z_{23}^{*}\right) \\
& +20 R_{N}^{3}\left(1-2 v_{N}\right)^{2} Z_{13}^{*}+16\left(4-5 v_{N}\right)\left(1-2 v_{N}\right) Z_{43}^{*}, \\
B_{N}^{*}(p)= & 3 R_{N}^{10}\left(1-2 v_{N}\right)\left(15 v_{N}-7\right) Z_{12}^{*}+60 R_{N}^{7}\left(v_{N}-3\right) v_{N} Z_{42}^{*}-24 R_{N}^{5}\left(1-2 v_{N}\right)\left(Z_{14}^{*}-7 Z_{23}^{*}\right) \\
& -40 R_{N}^{3}\left(1-2 v_{N}\right)^{2} Z_{13}^{*}-8\left(1-5 v_{N}\right)\left(1-2 v_{N}\right) Z_{43}^{*}, \\
C_{N}^{*}(p)= & -R_{N}^{10}\left(1-2 v_{N}\right)\left(7+5 v_{N}\right) Z_{12}^{*}+10 R_{N}^{7}\left(7-v_{N}^{2}\right) Z_{42}^{*}+12 R_{N}^{5}\left(1-2 v_{N}\right)\left(Z_{14}^{*}-7 Z_{23}^{*}\right) \\
& +20 R_{N}^{3}\left(1-2 v_{N}\right)^{2} Z_{13}^{*}-8\left(7-5 v_{N}\right)\left(1-2 v_{N}\right) Z_{43}^{*}
\end{aligned}
$$

with

$$
\begin{aligned}
Z_{\alpha \beta}^{*} & =L_{\alpha 1}^{(N-1) *} L_{\beta 2}^{(N-1) *}-L_{\beta 1}^{(N-1) *} L_{\alpha 2}^{(N-1) *}, \quad(\alpha, \beta) \in[1,4], \\
L^{(N-1) *} & =\prod_{i=1}^{N-1} M^{(i) *}
\end{aligned}
$$

and

$$
\begin{aligned}
& M^{(i) *}=\left[\begin{array}{cccc}
\frac{c_{i}}{3} & \frac{R_{i}^{2}\left(3 b_{i}-7 c_{i}\right)}{5\left(1-2 v_{i}\right)} & \frac{-12 \alpha_{i}}{R_{i}^{5}} & \frac{4\left(f_{i}-27 \alpha_{i}\right)}{15\left(1-2 v_{i}\right) R_{i}^{3}} \\
0 & \frac{\left(1-2 v_{i+1}\right) b_{i}}{7\left(1-2 v_{i}\right)} & \frac{-20\left(1-2 v_{i+1}\right) \alpha_{i}}{7 R_{i}^{7}} & \frac{-12 \alpha_{i}\left(1-2 v_{i+1}\right)}{7\left(1-2 v_{i}\right) R_{i}^{5}} \\
\frac{R_{i}^{5} \alpha_{i}}{2} & \frac{-R_{i}^{7}\left(2 a_{i}+147 \alpha_{i}\right)}{70\left(1-2 v_{i}\right)} & \frac{d_{i}}{7} & \frac{R_{i}^{2}\left(g_{i}+12 \alpha_{i}\left(7-10 v_{i+1}\right)-7 e_{i}\right)}{35\left(1-2 v_{i}\right)} \\
-\frac{5}{6}\left(1-2 v_{i+1}\right) \alpha_{i} R_{i}^{3} & \frac{7\left(1-2 v_{i+1}\right) \alpha_{i} R_{i}^{5}}{2\left(1-2 v_{i}\right)} & 0 & \frac{e_{i}\left(1-2 v_{i+1}\right)}{3\left(1-2 v_{i}\right)}
\end{array}\right], \\
& a_{i}=-\left(7-10 v_{i}\right)\left(7+5 v_{i+1}\right)\left(p+1 / t_{i}^{j+1}\right) \cdots\left(p+1 / t_{i}^{n_{i}}\right) N_{i+1}(p) \\
& +\left(7+5 v_{i}\right)\left(7-10 v_{i+1}\right)\left(p+1 / t_{i+1}^{j+1}\right) \cdots\left(p+1 / t_{i+1}^{n_{i+1}}\right) N_{i}(p), \\
& b_{i}=4\left(7-10 v_{i}\right)\left(p+1 / t_{i}^{j+1}\right) \cdots\left(p+1 / t_{i}^{n_{i}}\right) N_{i+1}(p)+\left(7+5 v_{i}\right)\left(p+1 / t_{i+1}^{j+1}\right) \cdots\left(p+1 / t_{i+1}^{n_{i+1}}\right) N_{i}(p) \text {, } \\
& c_{i}=\left(7-5 v_{i+1}\right)\left(p+1 / t_{i}^{j+1}\right) \cdots\left(p+1 / t_{i}^{n_{i}}\right) N_{i+1}(p)+2\left(4-5 v_{i+1}\right)\left(p+1 / t_{i+1}^{j+1}\right) \cdots\left(p+1 / t_{i+1}^{n_{i+1}}\right) N_{i}(p) \text {, } \\
& d_{i}=\left(7+5 v_{i+1}\right)\left(p+1 / t_{i}^{j+1}\right) \cdots\left(p+1 / t_{i}^{n_{i}}\right) N_{i+1}(p)+4\left(7-10 v_{i+1}\right)\left(p+1 / t_{i+1}^{j+1}\right) \cdots\left(p+1 / t_{i+1}^{n_{i+1}}\right) N_{i}(p) \text {, } \\
& e_{i}=2\left(4-5 v_{i}\right)\left(p+1 / t_{i}^{j+1}\right) \cdots\left(p+1 / t_{i}^{n_{i}}\right) N_{i+1}(p)+\left(7-5 v_{i}\right)\left(p+1 / t_{i+1}^{j+1}\right) \cdots\left(p+1 / t_{i+1}^{n_{i+1}}\right) N_{i}(p) \text {, } \\
& f_{i}=\left(4-5 v_{i}\right)\left(p+1 / t_{i}^{j+1}\right) \cdots\left(p+1 / t_{i}^{n_{i}}\right) N_{i+1}(p) \\
& -\left(4-5 v_{i+1}\right)\left(7-5 v_{k}\right)\left(p+1 / t_{i+1}^{j+1}\right) \cdots\left(p+1 / t_{i+1}^{n_{i+1}}\right) N_{i}(p), \\
& g_{i}^{*}=105\left(1-v_{i+1}\right)\left(p+1 / t_{i}^{j+1}\right) \cdots\left(p+1 / t_{i}^{n_{i}}\right) N_{i+1}(p) \text {, } \\
& \alpha_{i}=\left(p+1 / t_{i+1}^{j+1}\right) \cdots\left(p+1 / t_{i+1}^{n_{i+1}}\right) N_{i}(p)-\left(p+1 / t_{i}^{j+1}\right) \cdots\left(p+1 / t_{i}^{n_{i}}\right) N_{i+1}(p) \text {. }
\end{aligned}
$$

\section{- Bulk relation spectra}

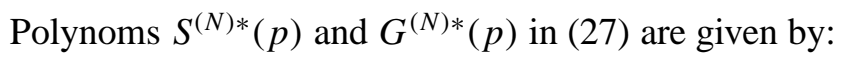

$$
Q^{(N-1) *}=\prod_{i=1}^{N-1} T_{i}^{*}\left(R_{i}\right),
$$




$$
\begin{aligned}
& T^{(i) *}\left(R_{i}\right)=\left[\begin{array}{cc}
2\left(\frac{1+v_{i}}{1-2 v_{i}}\right) 1_{i}^{*}+4 . h_{i}^{*} & \frac{4}{R_{i}^{3}}\left(h_{i}^{*}-1_{i}^{*}\right) \\
2 R_{i}^{3}\left(\left(\frac{1+v_{i+1}}{1-2 v_{i+1}}\right) h_{i}^{*}-\left(\frac{1+v_{i}}{1-2 v_{i}}\right) 1_{i}^{*}\right) & 2\left(\frac{1+v_{i+1}}{1-2 v_{i+1}}\right) h_{i}^{*}+4 . l_{i}^{*}
\end{array}\right], \\
& h_{i}^{*}=\left(p+1 / t_{i}^{j+1}\right) \cdots\left(p+1 / t_{i}^{n_{i}}\right) T_{i+1}(p), \quad S^{*}(p)=2 \frac{\left(1+v_{N}\right)}{\left(1-2 v_{N}\right)} R_{N}^{3} Q_{11}^{N-1 *}-4 Q_{21}^{N-1 *} \\
& l_{i}^{*}=\left(p+1 / t_{i+1}^{j+1}\right) \cdots\left(p+1 / t_{i+1}^{n_{i+1}}\right) T_{i}(p), \quad G^{*}(p)=3\left(R_{N}^{3} Q_{11}^{N-1 *}+Q_{21}^{N-1 *}\right) .
\end{aligned}
$$

\section{Appendix 3}

\section{$(N+1)$-phase model}

For the cylindrical $(N+1)$-phase model, coefficient $A_{N}^{*}(p), B_{N}^{*}(p)$ and $C_{N}^{*}(p)$ to be used in (20)-(25) are given by:

$$
\begin{gathered}
A_{N}^{*}(p)=\left(3-4 v_{N}\right) \cdot\left(Z_{41}^{*}+Z_{32}^{*}\right)-\left(3-4 v_{N}\right)^{2} Z_{13}^{*}+Z_{42}^{*}+Z_{43}^{*}-3 Z_{13}^{*}-3 Z_{12}^{*}, \\
B_{N}^{*}(p)=2 \cdot\left(\left(2 v_{N}-1\right) \cdot\left(Z_{41}^{*}+Z_{32}^{*}\right)-\left(3-4 v_{N}\right) Z_{13}^{*}-Z_{42}^{*}-Z_{43}^{*}+3 Z_{13}^{*}+3 Z_{12}^{*}\right), \\
C_{N}^{*}(p)=-Z_{41}^{*}-Z_{32}^{*}-Z_{13}^{*}+Z_{42}^{*}+Z_{43}^{*}-3 Z_{13}^{*}-3 Z_{12}^{*}, \\
Z_{\alpha \beta}^{*}=L_{\alpha 4}^{(N-1) *} L_{\beta 1}^{(N-1) *}-L_{\beta 4}^{(N-1) *} L_{\alpha 1}^{(N-1) *}, \quad(\alpha, \beta) \in[1,4], \\
L^{(N-1) *}=\prod_{i=1}^{N-1} M^{(i) *}
\end{gathered}
$$

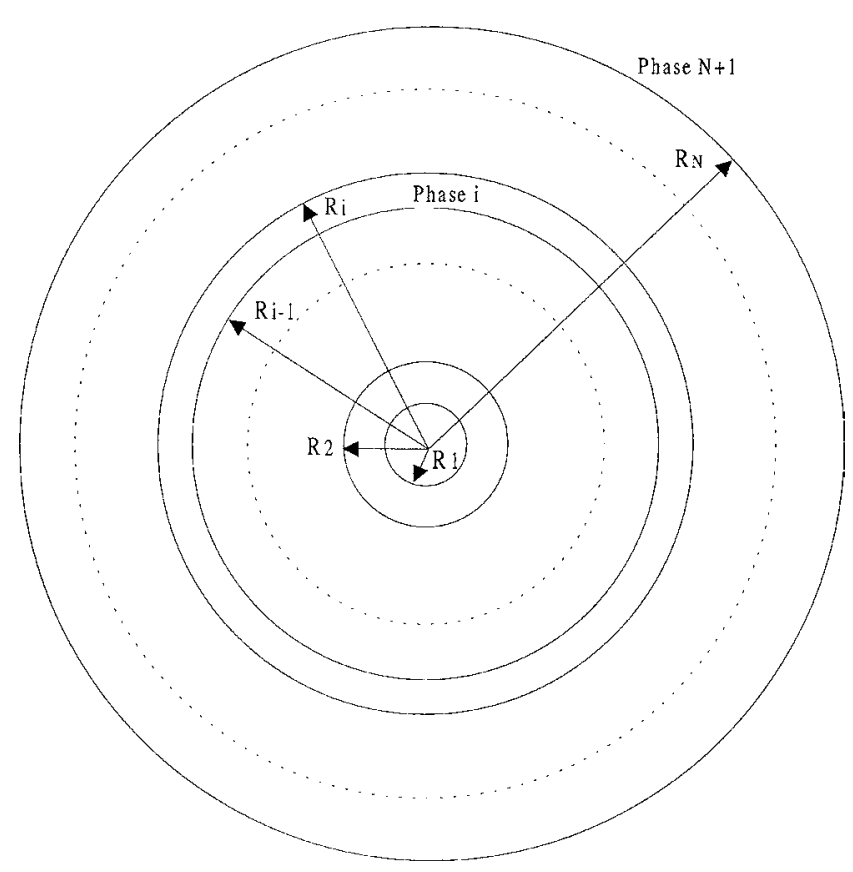

Figure A1. The $N$-layered spherical inclusion embedded in an infinite matrix. 
and

$$
\begin{aligned}
M^{(i) *}=\left[\begin{array}{cccc}
\frac{a_{i}}{q_{i}^{2}} & \frac{1}{q_{i}^{2}} \rho_{i} & \frac{1}{q_{k}^{2}} \rho_{i} & 0 \\
2 q_{i}^{4} b_{i} & q_{i}^{4} c_{i} & 2 q_{i}^{4} d_{i} & -q_{i}^{4} \rho_{i} \\
-3 q_{i}^{2} \rho_{i} & 0 & q_{i}^{2} a_{k} & q_{i}^{2} \rho_{i} \\
-6 d_{i} & 3 \rho_{i} & 2 b_{i} & c_{i}
\end{array}\right], \\
a_{i}=\left(p+1 / t_{i+1}^{j+1}\right) \cdots\left(p+1 / t_{i+1}^{n_{i+1}}\right) N_{i}(p)+\left(3-4 v_{i}\right)\left(p+1 / t_{i}^{j+1}\right) \cdots\left(p+1 / t_{i}^{n_{i}}\right) N_{i+1}(p), \\
b_{i}=\left(2 v_{k+1}-3\right)\left(p+1 / t_{i+1}^{j+1}\right) \cdots\left(p+1 / t_{i+1}^{n_{i+1}}\right) N_{i}(p)+\left(3-2 v_{k}\right)\left(p+1 / t_{i}^{j+1}\right) \cdots\left(p+1 / t_{i}^{n_{i}}\right) N_{i+1}(p), \\
c_{i}=\left(3-4 v_{k+1}\right)\left(p+1 / t_{i+1}^{j+1}\right) \cdots\left(p+1 / t_{i+1}^{n_{i+1}}\right) N_{i}(p)+\left(p+1 / t_{i}^{j+1}\right) \cdots\left(p+1 / t_{i}^{n_{i}}\right) N_{i+1}(p), \\
d_{i}=\left(1-2 v_{k+1}\right)\left(p+1 / t_{i+1}^{j+1}\right) \cdots\left(p+1 / t_{i+1}^{n_{i+1}}\right) N_{i}(p)+\left(2 v_{k}-1\right)\left(p+1 / t_{i}^{j+1}\right) \cdots\left(p+1 / t_{i}^{n_{i}}\right) N_{i+1}(p), \\
\rho_{i}=\left(p+1 / t_{i}^{j+1}\right) \cdots\left(p+1 / t_{i}^{n_{i}}\right) N_{i+1}(p)-\left(p+1 / t_{i+1}^{j+1}\right) \cdots\left(p+1 / t_{i+1}^{n_{i+1}}\right) N_{i}(p), \\
q_{i}=\frac{R_{i}}{R_{i+1}} .
\end{aligned}
$$

\section{Acknowledgements}

Yves Rougier and Claude Stolz are gratefully acknowledged for their initial contribution to this study.

\section{References}

Beurthey S., 1997. Modélisations du comportement d'alliages de polymères. PhD dissertation, École Polytechnique, Palaiseau, France.

Bornert M., Hervé É., Stolz C., Zaoui A., 1994. Self-consistent approaches and strain heterogeneities in two-phase elastoplastic materials. Appl. Mech. Rev. 47, S 66-76.

Bornert M., Stolz C., Zaoui A., 1996. Morphologically representative pattern-based bounding in elasticity. J. Mech. Phys. Solids 44, 307-331.

Bourgeois L., Hervé É., Rougier Y., 1996. Incompressible fibre-reinforced composites: Effective elastoplastic or viscoelastic behaviour. In: Markov K. (Ed.), Continuum Models and Discrete Systems, World Scientific, pp. 132-139.

Christensen R.M., Lo K.H., 1979. Solutions for effective shear properties in three-phase sphere and cylinder models. J. Mech. Phys. Solids 27, $315-330$

Hashin Z., 1962. The elastic moduli of heterogeneous materials. J. Appl. Mech. 29, 143-150.

Hershey A.V., 1954. The elasticity of an isotropic aggregate of anisotropic cubic crystals. J. Appl. Mech. 21, 236-240.

Hervé É., Zaoui A., 1990. Modelling the effective behaviour of nonlinear matrix inclusion composites. Eur. J. Mech. A/Solids 9, $505-515$.

Hervé É., Zaoui A., 1993. N-layered inclusion-based micromechanical modelling. Int. J. Engng. Sci. 31, 1-10.

Hervé É., Zaoui A., 1995. Elastic behaviour of multiply coated fibre-reinforced composites. Int. J. Engng. Sci. 33, 1419-1433.

Kröner E., 1977. Bounds for effective elastic moduli of disordered materials. J. Mech. Phys. Solids 25, 137-155.

Laws N., McLaughlin R.E., 1978. Self-consistent estimates for the viscoelastic creep compliance of composite materials. Proc. Roy. Soc. London A359, 251-273.

Mandel J., 1966. Mécanique des milieux continus. Gauthier-Villars, Paris.

Rougier Y., 1994. Étude du comportement sous irradiation: modélisation micromécanique de l'élastoviscoplasticité, PhD dissertation, École Polytechnique, Palaiseau, France.

Rougier Y., Stolz C., Zaoui A., 1993. Représentation spectrale en viscoélasticité linéaire des matériaux hétérogènes, C. R. Acad. Sci. Paris II 316, $1517-1522$.

Suquet P., 1987. Elements of homogenization for inelastic solid mechanics. In: Sanchez-Palencia E., Zaoui A. (Eds.), Homogenization Techniques for Composite Media, Springer-Verlag, Berlin, pp. 193-278.

Zaoui A., 1997. Structural morphology and constitutive behaviour of microheterogeneous materials. In: Suquet P. (Ed.), Continuum Micromechanics, Springer-Verlag, Wien, pp. 291-347. 